Canad. Math. Bull. Vol. 19 (2), 1976

\title{
ON SEMIPRIME AMPLE JORDAN RINGS
}

\author{
$J \subseteq H$ WITH CHAIN CONDITION ${ }^{(1)}$ \\ BY \\ DANIEL J. BRITTEN
}

The purpose of this paper is to point out that the arguments of [2] with slight modification extend the main result of [2] to the case of $H$ satisfying either $A C C$ or $D C C$ on quadratic ideals and they extend [6, Theorem 2] to $R$ being semiprime. Thus we obtain

THEOREM 1. Let $R$ be a semiprime associative ring with involution $*$ and $J a$ closed ample quadratic Jordan subring of $H(R)$ satisfying either ACC or DCC on quadratic ideals. Then $R$ is Goldie. In this case, $J$ has a Jordan ring of quotients $J^{\prime}$ which is a closed ample quadratic Jordan subring of $H\left(R^{\prime}\right)$ where $R^{\prime}$ is the associative ring of quotients of $R$.

In what follows, $R$ denotes an associative ring with involution $* ; H=$ $H(R)=\left\{x \in R: x^{*}=x\right\}: T=T(R)=\left\{x+x^{*}: x \in R\right\} ; \quad$ and $\quad N=N(R)=$ $\left\{x x^{*}: x \in R\right\}$. $J$ will denote a special quadratic Jordan ring; that is, $J$ will be an additive subgroup of $(R,+)$, closed under the quadratic operator $y U_{x}=x y x$ and the binary composition $x^{2}$. Following Montgomery [5], special quadratic Jordan subring $J$ of $H$ is called ample provided $N \cup T \subseteq J \subseteq H$.

Montgomery [5] and [6] removes the 2-torsion free assumption of Britten [1] and enlargens the class of Jordan subrings to be considered from $H$ to ample special quadratic Jordan subrings $J$. (If $\frac{1}{2} \in R$, then $H=T$ and so the only ample subring is $H$.) Thus for this paper, [2], [5] and [6] are the basic references.

In [5, Theorem 4.5], Montgomery assumes in addition to $J$ being ample that $x J x^{*} \subseteq J$ for all $x \in R$. This property is also valid for the Jordan ring of quotients obtained from this theorem and so we shall say that $J$ is closed ample if it is ample and $x J X^{*} \subseteq J$ for all $x \in R$.

Finally if $S \subseteq R, A_{\ell}(S)$ denotes $\{x \in R: x S=0\}$ and $A_{r}(S)$ denotes $\{x \in R: S x=0\}$, then we have

LEMMA 1. Let $R$ be a semiprime ring with *. Then

(i) if $B$ is a left ideal of $R$ and $A=A_{\ell}(B)$ then $B A=0$;

(ii) If $B$ is $a *$-ideal (i.e. $B^{*}=B$ ) of $R$ then $A=A_{\ell}(B)$ is $a{ }^{*}$-ideal and $B \cap(T \cup N)=0$ implies $B=0$.

Received by the editors June 2, 1975.

(1) Prepared while the author was at the 1974 SRI at the University of Calgary and held NRC Grant A-8471. Revised while the author was at the 1975 SRI at Dalhousie University. 
(i) is easily shown and (ii) is [5, Lemma 3.1]. We shall also have need of [5, Theorem 4.5] which we formulate as

THeOREM 2. Let $R$ be an associative ring with involution * and a ring of quotients $R^{\prime}$ ( $R^{\prime}$ has a natural involution extending *). Let $J$ be a closed ample Jordan subring of $H(R)$ such that every regular element of $J$ is regular in $R$. Then $J$ has a Jordan ring of quotients $J^{\prime}$ which is a closed ample Jordan subring of $H\left(R^{\prime}\right)$. When $J=H(R), J^{\prime}=H\left(R^{\prime}\right)$.

Proof. Since we are asking that $J^{\prime}$ be closed ample and Montgomery asked that it only be ample, we must check that $x J^{\prime} x^{*} \subseteq J^{\prime}$ for all $x \in R^{\prime}$. (Here we are using * to denote the natural involution on $R^{\prime}$ which extends the involution on $R$ given to us in the statement of the theorem.) Thus for us the ring of quotients $J^{\prime}$ exists, is an ample Jordan subring of $H\left(R^{\prime}\right)$ and $J^{\prime}=\left\{a U_{b}^{-1}: a, b \in J\right.$, $b$ regular in $J\}$.

Let $x \in R^{\prime}$ and $y \in J^{\prime}$. Express $x$ as $c^{-1} d$ and $y$ as $b^{-1} a b^{-1}$ where $d \in R, a \in J$ and $c, b$ are regular elements in $N$. Then $x y x^{*}=c^{-1} d b^{-1} a b^{-1} d^{*} c^{-1}$. By the common multiple property of $R$, we may express $d b^{-1}$ as $b_{1}^{-1} d_{1}$, with $b_{1} \in N$. Thus

$$
\begin{aligned}
x y x^{*} & =c^{-1} b_{1}^{-1} d_{1} a d_{1}^{*} b_{1}^{-1} c^{-1} \\
& =\left(d_{1} a d_{1}^{*}\right) U_{c U_{b 1}}^{-1} U_{b_{1}} .
\end{aligned}
$$

Set $s=\left(d_{1} a d_{1}^{*}\right) U_{c U_{b}}^{-1} \in J^{\prime}$ and $t=b_{1} \in J$. We have now reduced the problem to showing that $s U_{t} \in J^{\prime}$ for $s \in J^{\prime}$ and $t \in J$. This was done by Montgomery in her proof of [5, Theorem 4.5].

Let $R$ be a semiprime ring with * and $J$ an ample quadratic Jordan subring of $H$ satisfy either $A C C$ or $D C C$ on quadratic ideals.

For any subset $S \subseteq R$, we have $B=A_{\ell}(S)=A_{\ell} A_{r} A_{\ell}(S)$. In particular, if $S$ is $a^{*}$-ideal then $B$ is a ${ }^{*}$-ideal and $B$ is the left annihilator of the *-ideal $A=A_{r}(B)$. Let us gather together all such proper ${ }^{*}$-ideals $B$ into the set $\mathfrak{B}=\left\{B: B=A_{\ell}(A)\right.$ for ${ }^{*}$-ideal $A=A_{r}(B)$ with $\left.B \neq R\right\}$.

Since $B \cap J$ is a quadratic ideal of $J$ for all $B \in \mathfrak{B}$, if $J$ satisfies $A C C$ on quadratic ideals $B$ contains elements $B_{i}$ such that $B_{i} \cap J$ is not a proper subset of $B \cap J$ regardless of the choice of $B \in \mathfrak{B}$. That is, $\varnothing \neq \beta_{1}=\left\{B_{i} \in \mathfrak{B}: B_{i} \cap J\right.$ is maximal\}.

On the other hand, if $J$ satisfies $D C C$ on quadratic ideals, $B$ contains elements $B_{i}$ such that $A_{i} \cap J$ is minimal where $A_{i}=A_{r}\left(B_{i}\right)$. That is, $\varnothing \neq \beta_{2}=$ $\left\{B_{i} \in \mathfrak{B}: A_{i} \cap J\right.$ is minimal where $\left.A_{i}=A_{r}\left(B_{i}\right)\right\}$.

Now define

$$
\beta= \begin{cases}\beta_{1} & \text { if } J \text { satisfies } A C C \\ \beta_{2} & \text { if } J \text { satisfies } D C C\end{cases}
$$

LEMMA 2. Let $R$ be a semiprime ring with $*$ and $J$ an ample quadratic Jordan subring of $H$ satisfying either ACC or DCC on quadratic ideals. Then if $\beta$ is as 
above then

(i) $B_{i} \in \beta$ is $a^{*}$-prime ideal (i.e. $R / B_{i}$ is $a^{*}$-prime ring);

(ii) if $B_{i}$ and $B_{j}$ are distinct elements of $\beta$ then $B_{i} \nsubseteq B_{j}$ but $A_{\ell}\left(B_{i}\right) \subseteq B_{j}$;

(iii) $\beta$ is a finite set;

(iv) the elements of $\beta$ intersect at zero.

Proof. (i) Let $C$ and $D$ be nonzero *-ideals of $R$ such that $C D \subseteq B_{i} \in \beta$. Let $A_{i}=A_{r}\left(B_{i}\right)$.

First suppose that $J$ satisfies $A C C$ on quadratic ideals. If $D A_{i}=0$, then $D \subseteq B_{i}$. Therefore, we may assume that $A_{i} D+D A_{i} \neq 0$. By Lemma 1 (i), $B_{i} \cup C \subseteq A_{\ell}\left(A_{i} D+D A_{i}\right)$ and $A_{\ell}\left(A_{i} D+D A_{i}\right) \neq R$ be semi-primeness of $R$. But $B_{i} \cap J \subseteq A_{\ell}\left(A_{i} D+D A_{i}\right) \cap J$ and by maximality of $B_{i} \cap J$ we must have $B_{i} \cap J=$ $A_{\ell}\left(A_{i} D+D A_{i}\right) \cap J$ and hence $C \cap J \subseteq B_{i} \cap J$. Thus $\left(A_{i} C+C A_{i}\right) \cap J \subseteq A_{i} \cap B_{i}=$ 0 , by semi-primeness, and hence, by Lemma 1 (ii), $C A_{i}=0$ so that $C \subseteq B$.

Now suppose $J$ satisfies $D C C$ on quadratic ideals. If either $\left(A_{i} D+D A_{i}\right) \cap J$ or $\left(A_{i} C+C A_{i}\right) \cap J$ is zero, then we are done by Lemma 1 (ii). Thus we assume that neither is zero. By the minimality of $A_{i} \cap J$ we have

$$
\begin{aligned}
& 0 \neq\left(A_{i} D+D A_{i}\right) \cap J \subseteq A_{r}\left(C+B_{i}\right) \cap J=A_{i} \cap J \\
& 0 \neq\left(A_{i} C+C A_{i}\right) \cap J \subseteq A_{r}\left(D+B_{i}\right) \cap J=A_{i} \cap J
\end{aligned}
$$

Thus $A_{i} \cap J \subseteq A_{r}\left(C+D+B_{i}\right)$ and hence $Q=\left(A_{i} D+D A_{i}\right) \cap J$ is nonzero, by Lemma 1 (ii), and is such that $J U_{Q}=0$, contrary to the semi-primeness of $J$ given to us by [3, Theorem 2].

(ii) follows from the ${ }^{*}$-primeness of the elements $B_{i}$ of $\beta$. (See [2, Lemma 4]).

(iii) follows by showing that $\sum A_{i}$ is a direct sum and using the chain condition on $J$ in the presence of $0 \neq(N \cup T) \cap A_{i} \subseteq J$. (See [2, Lemma 5]).

(iv) Let $\left\{B_{1}, \ldots, B_{k}\right\}=\beta$ in accordance with (iii) and corresponding to each $B_{i} \in \beta$, let $A_{i}=A_{r}\left(B_{i}\right)$. Let $I=\bigcap_{1}^{k} B_{i}$ and suppose $I \neq 0$. Then $I A_{i}=0$ for $1 \leq i \leq k$ and by Lemma 1 (i), we have $A_{i} I=0$ so that $A_{i} \subseteq A_{\ell}(I)$ for each $i$. $A_{\ell}(I) \neq 0$ by the semi-primeness of $R$.

Assume that $J$ satisfies $A C C$ on quadratic ideals. Then there is some $j=1, \ldots, k$ such that $A_{\ell}(I) \cap J \subseteq B_{j} \cap J$. Set $Q=A_{j} \cap J \subseteq B_{j} \cap J$ and obtain the contradiction $J U_{Q}=0$.

Finally, assume that $J$ satisfies $D C C$. Since $A_{i} I=0$ for each $i=1, \ldots, k$, $I \subseteq A_{r}\left(\sum_{i}^{k} A_{i}\right)$. By the minimality of the $A_{i} \cap J$ 's there is some $1 \leq j \leq k$ such that $A_{r}(I) \cap J \supseteq A_{j} \cap J$. Set $Q=A_{j} \cap J$ and obtain $J U_{Q}=0$.

Froof of Theorem 1. By Lemma $2, R / B_{i}$ is an involution prime ring for each $B_{i} \in \beta$ where the involution on $R / B_{i}$ is determined by applying * to the representative of the coset. The homomorphic image of $J$ in $R / B_{i}$ is closed ample quadratic Jordan subring of $H\left(R / B_{i}\right)$ which satisfies either $A C C$ or $D C C$ 
accordingly as $J$ does. That is, $R / B_{i}$ satisfies the hypothesis of [6, Theorem 2] and hence $R / B_{i}$ is ${ }^{*}$-prime Goldie. Thus $R$ is semiprime Goldie. The last sentence of Theorem 1 follows from Theorem 2 after arguing, as Montgomery did in the proof of [5, Corollary 2], that regular elements of $J$ are regular in $R$.

Before closing we point out that the open question (stated in the closing remarks of [5]) as to the necessity of the common multiple property of a Jordan ring $J$ to have a ring of quotients may be answered negatively by considering the Jordan plus structure of the left Ore domain that is not a right Ore domain. However, the sufficiency of the common multiple property for $J$ to have a ring of quotients remains open.

\section{REFERENCES}

1. D. J. Britten, On Prime Jordan Rings H(R) with Chain Conditions, J. Algebra, 27 (1973), 414-421.

2. - On Semiprime Jordan Rings $H(R)$ with ACC, Proc. Amer. Math. Soc., 45 (1974), $175-178$.

3. T. S. Erickson and S. Montgomery, The Prime Radical in Special Jordan Rings, Trans. Amer. Math. Soc., 156 (1971), 155-164.

4. N. Jacobson, Structure of Rings, 2nd ed., Amer. Math. Soc. Colloq. Publ., Vol. 37, Amer. Math. Soc., Providence, R.I., 1968.

5. Susan Montgomery, Rings of Quotients for a Class of Special Jordan Rings, J. Algebra, 31 (1974), 154-165.

6. - Chain Condition on Symmetric Elements, Proc. Amer. Math. Soc. 46 (1974), 325-331.

DepartMent of Mathematics

UNIVERSITY OF WINDSOR,

WINDSOR, ONTARIO,

Canada 